\title{
The Approach of Cancer Related Fatigue in Rehabilitation Medicine: Part I - mechanisms, symptoms, clinical evaluation and screening.
}

\author{
Amalia Salca ${ }^{1}$, Alexandra Checiches ${ }^{1}$, Laszlo Irsay ${ }^{2}$ \\ ${ }^{1}$ - Rehabilitation Clinic, Cluj-Napoca \\ ${ }^{2}$ - Rehabilitation Clinic, Cluj-Napoca, University of Medicine and Pharmacy „Iuliu \\ Hatieganu”, Cluj-Napoca
}

\section{Abstract}

Cancer related fatigue (CRF) is the most disabling and distressing symptom in cancer, throughout the whole history of the illness, including successfully treated cases. Rehabilitation medicine could have a positive impact on these patients' outcomes, if well targeted. With these purpose, the rehabilitation clinician needs to correctly assess fatigue using standard, reliable scales. This review of articles and trials synthesizes the epidemiology, mechanics (including causes and effects), clinical presentation and means of assessment in CRF. CRF causes and mechanisms are not well known. Understanding them would provide specific targets to fatigue management. Clinical presentation is very variable, a wide range of features being found in literature. Poorly medical reports in assessing fatigue offer variable percentages of prevalence, inconsistent with patients' daily burden due to CRF. There are many tools used to assess fatigue in clinical research and on them depends the percentages reported as fatigue prevalence. The hereby gathered information are useful in the approach of a patient that addresses to a rehabilitation clinician for CRF management.

\section{Introduction}

Cancer related fatigue (CRF), known to be the most common and distressing symptom in cancer 1,2 , has a high prevalence across the cancer continuum (from the moment of the diagnosis through treatment and survivorship till end of life) ${ }^{3,4}$. Although is one of the most significant quality of life (QOL) issues in cancer care, it is not fully understood yet ${ }^{5}$.

The National Comprehensive Cancer Network (NCCN) definition of fatigue is a , persistent subjective sense of tiredness related to cancer treatment that interferes with usual functioning”. CRF differs from everyday life fatigue, which is temporary and is relieved by rest. It affects the quality of life through its sequel.

In multidisciplinary approach, rehabilitation medicine should address to quality of life improvement through interventions with functional impact. This can be done only based on a reliable baseline fatigue evaluation, detailed fatigue history, recordings of patients' comorbidities and treatments. Knowledge of precipitating factors and causes is neccessary for a good fatigue management.

\section{Epidemiology}

In literature there is a variability of fatigue prevalence, the reported rates ranging up to $99 \%{ }^{8}$. In patients with metastatic disease, the prevalence of cancerrelated fatigue exceeds $75 \%$, and cancer survivors report that fatigue is a disruptive symptom months or even years after treatment ${ }^{2}$.

All these numbers underestimate the real prevalence of CRF, proved by the lack of concordance between patient reports and medical records ${ }^{6}$. The later could be explained by the fact that the clinician is required to document only symptoms and signs relevant to the patient's cancer diagnose. Because of its high prevalence, CRF is a not only a major burden to the patient, but a substantial public health concern.

\section{Mechanisms}


The mechanisms of CRF are not yet fully understood, though many clinical studies have been conducted in this perspective. Among the early symptoms in patients with cancer, fatigue is usually exacerbated by the increased use of fatigueinducing multimodal treatments and dosedense, dose-intense protocols ${ }^{2}$.

The physiological determinants that enhance fatigue encompass a combination of metabolic, neurological and myofibrillar adaptations ${ }^{7}$. In cancer continuum, physical inactivity can also contribute to symptomatic fatigue, through deconditioning.

There are evidence of a genetic association between a functional promoter polymorphism in the IL-6 gene and severity of evening fatigue, morning fatigue, and sleep disturbance in oncology patients and their family care-givers ${ }^{8}$. Other studies show evidence of an association between fatigue and alterations ATP metabolism ${ }^{9}$. There are evidences of the following association: levels of inflammation, quality of life and survival ${ }^{3}$.

Studies of the Eastern Cooperative Oncology Group ${ }^{14}$ show that patient are very much bothered by symptom treatments, such as pain. This is explained by the frequent adverse effects that pain medication has. Eventually these patients are undertreated.

Last, but not least, CRF can be related to 5-HT dysregulation, vagal afferent activation, alterations in muscle and ATP metabolism, hypothalamic-pituitaryadrenal axis dysfunction, circadian rhythm disruption and cytokine dysregulation ${ }^{9}$.

Comorbidities should not be ignored when consulting a patient with cancer. They can as well contribute to CRF through organic insufficiencies or treatment adverse effects. Some of them are temporary, like infections, anemia, dehydration; others are chronic illnesses that should be carefully supervised. Most often associated with CRF in clinical practice are the following: anemia, depression, sleep disorders ${ }^{9}$.
According to research clinical studies, the factors most often implicated are the following: cancer treatment, pain, anemia, medications, cachexia/anorexia, metabolic disturbances, hormone deficiency or excess, psychological distress, physical deconditioning, sleep disturbances, excessive inactivity, pulmonary impairment, neuromuscular dysfunction, pain and other symptoms, pro-inflammatory cytokines, nutritional deficiencies, dehydration, infection, concomitant medical illness, cardiac impairment ${ }^{4,5,9}$.

\section{Clinical presentation of CRF}

Fatigue in cancer patients is considered a complex of several symptoms. In questionnairesused in clinical studies it is recorded either depending on the moment of the day as evening or morning fatigue, (highlighting sleep disturbances as well ${ }^{6}$ ), or by different scales.

Symptom -based research target the following issues: recognition, type of studied population, way of detection (documented, elicited or volunteered symptoms), temporal factors (onset, rhythmicity, and duration), severity, cause, comorbidities that should be assessed and outcomes ${ }^{10}$.

Fatigue is not well documented in medical reports ${ }^{6}$, though is the most common symptom in cancer patients; so recognition is a problem. The following complaints are often mentioned by patients: pain, nausea, difficulty sleeping, and gastrointestinal problems (diarrhea, constipation, irritable bowels). Patients describe fatigue as a sudden symptom, like „running into a brick wall”, or keeping them from accomplishing the things they want to do. Others stated that they were not able to do the kind of activities that they enjoyed doing.

Most studies mention CRF as a documented or a volunteered symptom, not an elicited one. Present from early phases of cancer continuum, this symptom is a burden for the patient all through treatment and afterwards, even among survivors. Its 
enhancement due to chemotherapy and radiotherapy is well known and research studies prove to be dependent on the severity prior to treatment ${ }^{11}$. The severity of fatigue can be assessed through a wide range of scales and index charts (see Clinical evaluation and screening).

Due to its sequel, fatigue is considered the most disabling cancer symptom and long term cancer history is associated with cognitive impairment, loss of physical abilities, low quality of life (QOL).

Comorbidities that are highly associated with fatigue are anemia, cardiac dysfunction, pulmonary impairment, history of depression, infection, weight loss. Some of these are curable, thus providing achievable goals to fatigue management.

\section{Clinical evaluation and screening}

The assessment method influences the percentage of patients reporting fatigue. Variations of its prevalence appears even with comparable patient cohorts and similar assessment scales ${ }^{5}$. For CRF there is a wide range of scales, inventories of its features, severity and interferences with functioning. Most of them are used in literature to classify fatigue into 3 categories: mild, moderate and severe. The numbers of the scales are used as cut-points for these categories.

Some clinical longitudinal trials use different tools along the evolution of the study, or employ the same one upon enrollment (baseline fatigue), in the followup phases and at the end.

Piper Fatigue Scale (PFS) is a 22item , self-report scale, that measures 4 dimensions of subjective fatigue (behavioral/severity, sensory, cognitive/mood, affective meaning) ${ }^{1}$. The severity of the fatigue can be point out through scores, like Lee Fatigue Scale (LFS), which includes 13 items ${ }^{8}$.
The Edmonton Symptom Assessment System (ESAS) is a good tool for screening the advanced cancerpopulation ${ }^{12}$, while its revised version, the ESAS-r, is easier for patients to understand 13. The BFI (Brief Fatigue Inventory) is a reliable instrument for quick assessment offatigue level in cancer patients and highlights those with severe fatigue. ${ }^{16}$

MDASI(MD Anderson Cancer Center Symptom Inventory is a validated measure that is similar to the Brief Fatigue Inventory from the point of view of structure and patient burden assessment ${ }^{11}$.

The Karnofsky Performance Scale Index (KPSI) allows patients to be classified as to their functional impairment. It is used to compare effectiveness of different therapies and to assess the prognosis in individual patients. The 21item General Sleep Disturbances Scale (GSDS) evaluates various aspects of sleep disturbances, rating them on a scale from 0 (never) to 7 (every day).

Functional Assessment of cancer Therapy - General (FACT-G) is a 27-item questionnaire that is enabled to measure Quality of life on four subscales: physical well-being (7 items), social well-being,

As one can see, evaluating CRF involves the patients' subjective perception, so symptom assessment has a documentation and volunteered feature rather than an elicited one. There are studies that go further than this and address specifically to self-management, meaning self-assessment, for the initial part of the programs. An example is RESTORE, which is an exploratory trial of an online intervention to enhance self-efficacy to manage problems associated with CRF following primary cancer treatment ${ }^{16}$. 
Table 1: KARNOFSKY PERFORMANCE STATUS SCALE DEFINITIONS RATING (\%) CRITERIA

\begin{tabular}{|c|c|}
\hline \multirow{3}{*}{$\begin{array}{l}\text { Able to carry on normal activity and to work; no } \\
\text { special care needed. }\end{array}$} & $\begin{array}{l}100=\text { Normal no complaints; no evidence } \\
\text { of disease. }\end{array}$ \\
\hline & $\begin{array}{l}90=\text { Able to carry on normal activity; } \\
\text { minor signs or symptoms of disease. }\end{array}$ \\
\hline & $\begin{array}{l}\text { 80=Normal activity with effort; some } \\
\text { signs or symptoms of disease. }\end{array}$ \\
\hline \multirow{3}{*}{$\begin{array}{l}\text { Unable to work; able to live at home and care for } \\
\text { most personal needs; varying amount of assistance } \\
\text { needed. }\end{array}$} & $\begin{array}{l}70=\text { Cares for self; unable to carry on } \\
\text { normal activity or to do active work. }\end{array}$ \\
\hline & $\begin{array}{l}60=\text { Requires occasional assistance, but is } \\
\text { able to care for most of his personal needs. }\end{array}$ \\
\hline & $\begin{array}{l}50=\text { Requires considerable assistance and } \\
\text { frequent medical care. }\end{array}$ \\
\hline \multirow{5}{*}{$\begin{array}{l}\text { Unable to care for self; requires equivalent of } \\
\text { institutional or hospital care; disease may be } \\
\text { progressing rapidly. }\end{array}$} & $\begin{array}{l}\text { 40=Disabled; requires special care and } \\
\text { assistance. }\end{array}$ \\
\hline & $\begin{array}{l}\text { 30=Severely disabled; hospital admission } \\
\text { is indicated although death not imminent. }\end{array}$ \\
\hline & $\begin{array}{|lllr|}20=\text { Very } & \text { sick; } & \text { hospital } & \text { admission } \\
\text { necessary; } & \text { active } & \text { supportive } & \text { treatment } \\
\text { necessary. } & & & \\
\end{array}$ \\
\hline & $\begin{array}{l}\text { 10=Moribund; fatal processes progressing } \\
\text { rapidly. }\end{array}$ \\
\hline & $0=$ Dead \\
\hline
\end{tabular}


Though there is a wide range of scales and inventory tools, in the literature there are many investigator- developed scales, designed according to the objectives of certain studies.

\section{Discussions}

Common barriers in reporting fatigue relate to patient (personal or cultural beliefs), professionals (lack of documentation on the topic) and institutions (lack of supportive care referrals). The system barriers encountered relate to assessment and management, because their documentation is not a common requirement in most health care institutions. A frequent patient-related barrier is the belief that the doctor would ask about fatigue if it was important ${ }^{1}$. Thus prevention of fatigue enhancement is hard to achieve in clinical practice. PatientReported Outcomes (PRO) such as patientreported symptoms are used in research, but are not yet used in clinical practice.

The Quality Oncology Practice Initiative (QOPI), proposed by the American Society of Clinical Oncology, stresses documentation of all symptoms as part of symptom management and therefore it might increase the motivation for greater concordance between patients' symptoms and medical records. On the other hand any research made on this subject could inform and facilitate its implementation ${ }^{6}$.

Most studies neglect whether fatigue relates to the primary illness or to the reduced physical activity ${ }^{7}$. Knowledge of the skeletal muscle adaptations induced by physical inactivity and their contribution to fatigue symptoms is important for the utility of exercise rehabilitation as an intervention to alleviate the physiological precipitating factors of fatigue ${ }^{7}$. The quality of life in cancer patients has a strong influence on their survival and it is influenced by fatigue, pain and sleep disturbances.

Some of the precipitating factors of fatigue are treatable, but on the other hand their therapy has adverse side effects that bother the patient, according to literature 11. Such an example would be pain treatment ${ }^{14}$, which makes the management of fatigue more difficult.

Taking into considerations how much patients beliefs influence their reports and communication with health care professionals, educational programs could improve the interactions between patients and healthcare providers. This would facilitate the implementation of assessment guidelines regarding $\mathrm{CRF}^{1}$.

When approaching a patient who has been diagnosed with cancer, rehabilitation professionals should bear in mind that recognition, baseline evaluation, comorbidities documentation and periodical ambulatory consultations are crucial in CRF management. Patients might underestimate this symptom, when addressing to medical care facilities, though is affecting they daily activity. Therefore, detailed questionnaires should be employed in rehabilitation institutions dealing with kind of cases.

Rehabilitation Medicine has among main objectives the evaluation of functionality in various diseases. Patients should be asked about their perception of the physical, emotional and cognitive tiredness or exhaustion related to cancer and how it interferes with their daily activities. The lack of functionality can be documented or elicited by means of various test that simulate everyday life activities. Rehabilitation professionals can apply muscle and performance testing in these patients to objectify their complaints. From early phases of interactions, they can interfere through education, a component that has been proofed to improve patients' way of addressing to medical care givers and clinicians. 


\section{Conclusions}

All the patient related barriers in assessing fatigue create an imperative for professionals to take aggressive action in assessing and treating fatigue. Fatigue is a complex complaint among cancer patient along their history, including survivors. Its etiology is multifactorial and both clinical expression and sequel have a high grade of individual variability.

Some of the precipitating factors of fatigue can be controlled through therapy. CRF is described through a wide range of complaints among patients. BFI, ESAS are wide used tools for fatigue assessments, but literature offers many others that stress different features of the symptoms.

Motivation of reporting fatigue in medical papers should be increased. The effects of fatigue have a major impact on patients' quality of life. Both issues would benefit from educational programs among patients and caregivers.

There are many clinical research done in the field of CRF, but still little is known about causes, symptoms and predictors. Further studies are neccessary on this type of population to understand the features of fatigue at different severity grades and thus providing specific objectives for the management of CRF.

Rehabilitation medicine can have a major impact on CRF outcome if well conducted through targeted documentation, utter evaluation, periodic enhancement screening. All of these will provide realistic objectives for the rehabilitation therapeutic program. The latter will be discussed in the second part of this paper that is due to appear in the next issue of the Journal. Its indications, contraindications, components and effectiveness will be detailed, according to guidelines and recent articles.

\section{References}

1. Borneman T, Piper BF, Sun VC, Koczywas M, Uman G, Ferrell B. Implementing the Fatigue Guidelines at one NCCN member institution: process and outcomes. J Natl ComprCancNetw. 2007 Nov; 5(10):1092-101.

Available at: http://www.ncbi.nlm.nih.gov/pmc/articles/PM C2515169/

2. Mock $\mathrm{V}^{1}$, Atkinson A, Barsevick AM, Berger AM, Cimprich B, Eisenberger MA, Hinds P, Kaldor P, Otis-Green SA, Piper $\underline{B F}$. Cancer-related fatigue. Clinical Practice Guidelines in Oncology. J Natl Compr Canc Netw. 2007 Nov; 5(10):105478.http://www.jnccn.org/content/5/10/1054.lon g

3. Bock PR, Hanisch J, Matthes $H$, Zänker KS. Targeting inflammation incancerrelated-fatigue: a rationale for mistletoe therapy as supportive care in colorectal cancer patients. Inflamm Allergy Drug Targets. 2014; 13(2):105-11.

Available at: http://www.ncbi.nlm.nih.gov/pmc/articles/PM C4133960/

$4 . \quad$ Mitchell SA. Cancer-related fatigue: state of the science. PM R. 2010 May; 2(5):364-

83.http://www.pmrjournal.org/article/S19341482\%2810\%2900292-3/pdf

5. Wang XS, Zhao F, Fisch MJ, O'Mara AM, Cella D, Mendoza TR, Cleeland CS. Prevalence and characteristics of moderate to severe fatigue: a multicenter study in cancer patients and survivors. Cancer. 2014 Feb 1; 120(3):425-32.

Available at: http://onlinelibrary.wiley.com/doi/10.1002/cncr $.28434 /$ full

6. Sikorskii A, Wyatt G, Tamkus D, Victorson D, Rahbar MH, Ahn S. Concordance between patient reports of cancer-related symptoms and medical records documentation. J Pain Symptom Manage. 2012 Sep; 44(3):362-72.

Available at: http://www.ncbi.nlm.nih.gov/pmc/articles/PM C3432740/

7. Maughan D, Toth M. Discerning Primary and Secondary Factors Responsible for Clinical Fatigue in Multisystem Diseases. Biology. 3(3): 606-622. 
Available at: http://www.mdpi.com/20797737/3/3/606

8 . Miaskowski C, Dodd $\mathrm{M}$, Lee $\mathrm{K}$, West C, Paul SM, Cooper BA, Wara W, Swift PS, Dunn LB, Aouizerat BE. Preliminary evidence of an association between a functional interleukin-6 polymorphism and fatigue and sleep disturbance in oncology patients and their family caregivers. J Pain Symptom Manage. 2010 Oct; 40(4):531-44.

Available at: http://www.ncbi.nlm.nih.gov/pmc/articles/PM $\underline{\mathrm{C} 2952712 /}$

9. Ryan JL, Carroll JK, Ryan EP, Mustian KM, Fiscella K, Morrow GR. Mechanisms of cancer-related fatigue. Oncologist. 2007; 12 Suppl 1:22-34.

Available at: http://theoncologist.alphamedpress.org/content 112/suppl 1/22.long

10. Kroenke K. Studying symptoms: sampling and measurement issues. Ann Intern Med. 2001 May 1; 134(9 Pt 2):844-53. http://annals.org/article.aspx?articleid=714510 \&resultClick $=3$

11. Fisch MJ, Zhao F, O'Mara AM, Wang XS, Cella D, Cleeland CS. Predictors of significant worsening of patient-reported fatigue over a 1-month timeframe in ambulatory patients with common solid tumors. Cancer. 2014 Feb 1; 120(3):442-50.

Available

at:

http://onlinelibrary.wiley.com/doi/10.1002/cncr $.28437 /$ abstract

12. Zeng L, Koo K, Zhang L, Jon F, Dennis K, Holden L, Nguyen J, Tsao M, Barnes E, Danjoux C, Sahgal A, Chow E. Fatigue in advanced cancer patients attending an outpatient palliative radiotherapy clinic as screened by the Edmonton Symptom Assessment System. Support Care Cancer. $2012 \quad$ May; 20(5):103742.http://link.springer.com/article/10.1007\%2F s00520-011-1179-8

13. Watanabe SM ${ }^{1}$, Nekolaichuk CL, Beaumont C. The Edmonton Symptom Assessment System, a proposed tool for distress screening in cancer patients: development and refinement. Psychooncology. 2012 Sep; 21(9):97785.http://www.ncbi.nlm.nih.gov/pubmed/?term =Watanabe+SM1\%2C+Nekolaichuk+CL\%2C +Beaumont+C.+The+Edmonton+Symptom $+\mathrm{A}$ ssessment + System $\% 2 \mathrm{C}+\mathrm{a}+$ proposed + tool + for
+ distress+screening + in + cancer+patients $\% 3 \mathrm{~A}+$ development+and+refinement.+Psychooncolo gy.+2012+Sep\%3B+21\%289\%29\%3A977-85.

14. Zhao $F^{1}$, Chang VT, Cleeland

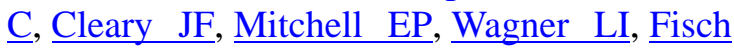
MJ. Determinants of pain severity changes in ambulatory patients with cancer: an analysis from Eastern CooperativeOncology Group trial E2Z02. J Clin Oncol. 2014 Feb 1; 32(4):3129.http://jco.ascopubs.org/content/32/4/312.long 15. Oxford Textbook of Palliative Medicine, Oxford University Press. 1993; 109. 16. Grimmett C, Armes J, Breckons M, Calman L, Corner J, Fenlon D, Hulme C, May CM, May CR, Ream E, Richardson A, Smith PW, Yardley L, Foster C. RESTORE: an exploratory trial of an online intervention to enhance self-efficacy to manage problems associated with cancer-related fatigue following primary cancer treatment: study protocol for a randomized controlled trial. Trials. 2013 Jun 21; 14:184.

Available

at: http://www.trialsjournal.com/content/14/1/184 17. Mendoza TR, Wang XS, Cleeland CS, Morrissey M, Johnson BA, Wendt JK, Huber SL. The rapid assessment of fatigue severity in cancer patients: use of the Brief Fatigue Inventory. Cancer. 1999 Mar 1;85(5):1186-96. Cancer-related fatigue. Clinical Practice Guidelines in Oncology.http://www.ncbi.nlm.nih.gov/pubme $\mathrm{d} /$ ?term=Mendoza+TR\%2C+Wang $+\mathrm{XS} \% 2 \mathrm{C}+$ Cleeland+CS\%2C+Morrissey+M\%2C+Johnso n+BA\%2C+Wendt+JK\%2C+Huber+SL.+The + rapid+assessment+of+fatigue+severity + in + ca ncer+patients $\% 3 \mathrm{~A}+$ use + of + the + Brief + Fatigue +Inventory.+Cancer.+1999+Mar+1\%3B85\%28 5\%29\%3A1186-96 